\title{
Unscreened: Urgent and Emergent Surgical Outcomes in the Early COVID-19 Pandemic
}

Christina Colosimo ${ }^{1}$, Jason Kelly ${ }^{2}$, James Coker ${ }^{1}$, Sidra Bhuller ${ }^{1}$, Eric Ballman ${ }^{1}$, Christina Baker-Sparr ${ }^{3}$, James Yon ${ }^{4}$, Brendon Cornett ${ }^{5}$, Oliwier Dziadkowiec ${ }^{5}$, John Weaver ${ }^{1}$

1. General Surgery, HCA HealthONE - Sky Ridge Medical Center, Lone Tree, USA 2. Administration, HCA HealthONE Sky Ridge Medical Center, Lone Tree, USA 3. Clinical Data Analytics, HCA HealthONE - Sky Ridge Medical Center, Lone Tree, USA 4. General Surgery, HCA HealthONE - Swedish Medical Center, Englewood, USA 5. Graduate Medical Education, HCA Healthcare, Lone Tree, USA

Corresponding author: Christina Colosimo, chris.colosimo@gmail.com

\section{Abstract}

\section{Background}

Creating useful recommendations for changes in surgical protocols during the severe acute respiratory syndrome coronavirus 2 (SARS-CoV-2) pandemic has been difficult due to a lack of studies based on representative samples. This study evaluates the clinical outcomes and characteristics of patients undergoing urgent or emergent surgeries.

\section{Methods}

This is a multi-center (eight-hospital), retrospective, observational study of urgent and emergent surgical patients from Colorado and Kansas, the United States, in the early stages of the SARS-CoV-2 pandemic. Patient groups were divided based on their coronavirus disease 2019 (COVID-19) status: positive, negative and untested. COVID-19 testing was performed after the surgery if patients were symptomatic.

\section{Results}

The analysis includes 5,547 patients who underwent surgery from March 1, 2020 to May 17, 2020. Seventyfour percent $(4,096)$ were not tested for COVID-19 due to lack of symptoms. Out of the 1,451 tested patients, 1,412 tested negative, and 39 tested positive. Out of all the patients who tested positive, $69.23 \%$ were admitted to the intensive care unit (ICU), whereas $16.72 \%$ of untested and $21.25 \%$ of the negative patients. The invasive ventilation rate for the patients that tested positive was $46.15 \%, 4.22 \%$ for untested, and $8.85 \%$ for patients who tested negative. The mortality rate in the positive group was $7.69 \%, 1.10 \%$ in the untested group, and $1.56 \%$ in the positive group.

Review began 11/18/2020 Review ended 11/28/2020 Published 12/03/2020

\section{๑) Copyright 2020}

Colosimo et al. This is an open access article distributed under the terms of the Creative Commons Attribution License CC-BY 4.0., which permits unrestricted use, distribution, and reproduction in any medium, provided the original author and source are credited.

\section{Conclusion}

Patients who tested positive for COVID-19 had worse clinical outcomes than patients who tested negative and untested. We recommend creating criteria for testing based on patient characteristics and surgical procedure rather than testing all patients awaiting surgery; this would allow us to conserve resources moving forward.

Categories: General Surgery, Public Health, Environmental Health

Keywords: covid-19, sars-cov-2 pandemic, testing, urgent surgeries, emergent surgeries

\section{Introduction}

Clinical decision making during the severe acute respiratory syndrome coronavirus 2 (SARS-CoV-2; the causative virus of coronavirus disease 2019 [COVID-19]) global pandemic has been extremely challenging due to the possible transmission of the virus through asymptomatic carriers and lack of data regarding postsurgical clinical outcomes for COVID-19 positive patients [1, 2]. Studies published during the pandemic created a proliferation of anecdotal evidence regarding outcomes of COVID-19 positive patients [2, 3]. These smaller, underpowered studies have led to broad clinical recommendations and unproven changes in surgical protocols. Performing surgery on asymptomatic patients who have not been tested for COVID-19 with no outcomes data is one prominent example [4].

The American Society of Anesthesiologists' statement on perioperative testing notes that "Patients who are infected with SARS-CoV-2, the virus responsible for the COVID-19 disease, have higher perioperative morbidity and mortality" [5]. The long incubation period for the SARS-CoV-2 virus has raised concerns that patients may undergo surgery during this infected but asymptomatic phase [6, 7]. Therefore, universal testing efforts and protocols have been created to help alleviate concerns of patient and surgical staff safety 
Some of the current recommendations are based on three small retrospective studies $[4,9,10]$. Aminian et al. reported a 50\% mortality rate in four COVID-19 positive patients who underwent urgent surgery in Iran. Two of the four patients were readmitted with COVID-19 approximately two weeks postoperatively, raising the possibility that they may not have been infected at the time of surgery. The third patient was scheduled for surgery but became symptomatic prior to surgery and was never in an operating room [9].

Lei et al. identified 34 operative patients with confirmed COVID-19, of which 15 (44.1\%) required intensive care unit (ICU) care and had an overall mortality rate of $20.5 \%$ [4]. The authors compared the mortality rate of the 34 surgical patients to the overall mortality rate of COVID-19 positive patients. This was comparing a population with potentially less disease burden to surgical patients. The paper also does not describe the mortality rate for non-surgical COVID-19 patients in their facility. Thus, the paper was not able to compare the mortality rates of surgical and non-surgical patients, making it impossible to claim a higher mortality rate in COVID-19 positive patients who underwent surgery. The study also suggested that surgery accelerates the symptoms of COVID-19 by examining median time from surgery to symptoms but does not take into account that patients had been admitted to the hospital for a median of two-and-a-half days prior to surgery. Including those extra two-and-a-half days, the median time to first symptoms suggests that patients were infected on the day of admission and had the same median five days from infection to onset of symptoms as reported elsewhere in China. Since we do not know when the patients were infected, we do not know if the symptoms were accelerated, and they could have been infected in the hospital on day one.

The only study that examined patient outcomes from patients who tested positive for COVID-19 and underwent surgery that was available at the time this manuscript was written came from the COVIDSurg Collaborative on May 29, 2020. The authors reviewed data from 1,128 patients from 235 hospitals in 24 countries that underwent emergency or elective surgeries between January 1, 2020 and March 31, 2020. The authors found the 30 -day mortality rate to be $23.8 \%$, with pulmonary complications occurring in $51.2 \%$ [11]. The patient demographics affecting the mortality rate in the paper included male gender and age 70 years or older. The COVIDSurg Collaborative study also did not include a comparison of unscreened patients to patients testing negative and surgical outcomes. The paper does not provide a representative emergency or elective surgery sample from each of the 235 hospitals.

As elective surgical procedures resume in healthcare systems in the United States and worldwide, more highquality information is needed to address both patient and medical provider concerns. This information would also help redefine the guidelines and recommendations for patient care in the COVID-19 era.

Our study evaluates 5,547 patients undergoing urgent or emergent surgeries in eight hospitals in Denver, Colorado, and Wichita, Kansas, which are part of a large healthcare system. Outcomes of this study have the potential to generate insights on resuming elective surgical cases and to quantify the benefit of perioperative testing.

\section{Materials And Methods \\ Study design and participants}

This is a multi-center, retrospective, observational study within one healthcare system in Colorado (six hospitals total) and Kansas (two hospitals total), located in the United States in the early stages of the SARSCoV-2 pandemic. The study examined 5,547 patients who underwent surgery from March 1, 2020 to May 17, 2020. All patients that underwent surgery in the eight facilities were included in the study. During this time, all eight hospitals had the same policy to put a hold on elective surgery and only allow urgent and emergent cases. All patient data was de-identified by the quality and patient safety data manager before analysis. IRB exemption was obtained for the study.

The patient population consisted of urgent or emergent surgical patients in one regional healthcare system. Patient groups were divided based on their COVID-19 status: positive, negative, and untested. Data was collected from a de-identified Electronic Health Record (EHR) during the early stages of the SARS-CoV-2 (COVID-19) pandemic in Colorado and Kansas in the United States. COVID-19 testing was not performed prior to surgery, and all symptoms or complications related to the infection became apparent postoperatively.

\section{Statistical analysis}

Due to large differences in sample sizes, particularly with the smaller COVID-19 positive group, Cramér's V was used to utilize effect size in addition to the $p$-value as our measure of statistical differences between groups following the Chi-Square test. Cramér's V describes the magnitude of the difference, rather than just whether the likelihood of the difference being observed was by chance (p-value). Effect size cut-off values (Table 1) for less than small, small and medium used Cohen's recommendation with two degrees of freedom [12]. 


\section{Cureus}

\begin{tabular}{|l|l|l|l|}
\hline Degree of freedom & Small & Medium & Large \\
\hline 1 & 0.10 & 0.30 & 0.50 \\
\hline 2 & 0.07 & 0.21 & 0.35 \\
\hline
\end{tabular}

TABLE 1: Effect size cut-off values

Kruskal-Wallis rank test was used to compare medians among the groups for continuous variables because they were not normally distributed. These normality violations are entirely consistent with variables such as length of stay (LOS) and physiological characteristics. As with the Chi-Squared test, we utilized effect size (Eta-squared) to describe the between-group differences rather than p-value.

Holm-Bonferroni p-value adjustment method was used to penalize/adjust the p-value for multiple comparisons [13]. Additionally, alpha level was set to 0.01 in order to further protect from falsely rejecting the null-hypothesis with a large sample size.

\section{Outcome measures}

Three groups (untested, COVID-19 positive, and COVID-19 negative patients) were compared based on their demographic characteristics and primary surgeries, bedside procedures, ICU usage, comorbidities, medication use, symptoms, discharge and LOS, and complications.

\section{Results}

During the first COVID-19 peak between March 1, 2020 and May 17, 2020, 5,547 patients underwent urgent or emergent procedures in a total of eight hospitals located in Denver, Colorado, and Wichita, Kansas. Of those patients, 1,451 (26\%) were tested for COVID-19, and 39 (2.7\%) had positive test results.

No meaningful differences in median age were noted between the three groups (Table 2). More females were found in the untested (54.5\%) and negative (52.4\%) groups, and more males in the positive group (61.5\%). All of the patients' operations were filed under the surgical subspecialty of their primary surgery. Listed below are the percentages of each group who underwent surgery in that surgical subspecialty. The majority of the patients had either general (33.99\%) or orthopedic surgery (22.34\%). We defined general surgery as general acute care surgery (appendectomies, cholecystectomies, herniorrhaphy, etc.) and colorectal (colon and anorectal surgeries). There were significant differences between all three groups regarding the percentage of general surgeries ( $\mathrm{p}<0.01$ ), with $53.85 \%$ of the positive patients undergoing a general surgical procedure. A significantly higher proportion of patients in the negative group underwent neurosurgery $(\mathrm{p}<$ 0.01). Ophthalmology recorded only four surgeries, of which all patients were in the untested group. Although there are statistically significant percentage differences between the three groups, the magnitude of these differences can be described as "small". 


\section{Cureus}

\begin{tabular}{|c|c|c|c|c|c|c|}
\hline & Untested ( $n=4096$ ) & Negative $(n=1412)$ & Positive ( $n=39$ ) & P-values & Cramer's V & Effect size \\
\hline Age & & & & 1.00 & $0.001^{\ddagger}$ & $<$ Small \\
\hline Median (IQR) & $60(33-87)$ & $61(38-84)$ & $62(37-87)$ & & & \\
\hline Sex & & & & 1.00 & 0.023 & $<$ Small \\
\hline Female & $2233(54.53 \%)$ & $740(52.41 \%)$ & $15(38.46 \%)$ & & & \\
\hline Male & $1862(45.47 \%)$ & $672(47.59 \%)$ & $24(61.54 \%)$ & & & \\
\hline \multicolumn{7}{|l|}{ Primary surgery } \\
\hline Cardio-thoracic & $106(2.59 \%$ & $54(3.82 \%)$ & $0(0 \%)$ & 1.00 & 0.035 & $<$ Small \\
\hline Colorectal & $261(6.37 \%)$ & $73(5.17 \%)$ & $2(5.13 \%)$ & 1.00 & 0.022 & $<$ Small \\
\hline Endocrine & $31(0.76 \%)$ & $8(0.57 \%)$ & $0(0 \%)$ & 1.00 & 0.012 & $<$ Small \\
\hline Endoscopy & $236(5.76 \%)$ & $61(4.32 \%)$ & $2(5.13 \%)$ & 1.00 & 0.028 & $<$ Small \\
\hline ENT & $23(0.56 \%)$ & $15(1.06 \%)$ & $0(0 \%)$ & 1.00 & 0.027 & $<$ Small \\
\hline General & $1332(32.52 \%)$ & $366(25.92 \%)$ & $21(53.85 \%)$ & $<0.01$ & 0.075 & $=$ Small \\
\hline Neurosurgery & $548(13.38 \%)$ & $283(20.04 \%)$ & $1(2.56 \%)$ & $<0.01$ & 0.086 & $=$ Small \\
\hline OBGYN & 237 (5.45\%) & 73 (5.45\%) & $2(5.13 \%)$ & 1.00 & 0.007 & $<$ Small \\
\hline OMFS & $19(0.46 \%)$ & $4(0.28 \%)$ & $1(2.56 \%)$ & 1.00 & 0.030 & $<$ Small \\
\hline Ophthalmology & $4(0.10 \%)$ & $0(0 \%)$ & $0(0 \%)$ & & & \\
\hline Orthopedic & $885(21.61 \%)$ & $348(24.65 \%)$ & $6(15.38 \%)$ & 1.00 & 0.035 & $<$ Small \\
\hline Plastic & $51(1.25 \%)$ & $24(1.70 \%)$ & $1(2.56 \%)$ & 1.00 & 0.019 & $<$ Small \\
\hline Transplants & $6(0.15 \%)$ & $9(0.64 \%)$ & $0(0 \%)$ & 0.86 & 0.041 & $<$ Small \\
\hline Urology & $285(6.96 \%)$ & $72(5.10 \%)$ & $1(2.56 \%)$ & 1.00 & 0.036 & $<$ Small \\
\hline Vascular & $72(1 . / 6 \%)$ & $18(1.27 \%)$ & $2(5.13 \%)$ & 1.00 & 0.028 & $<$ Small \\
\hline
\end{tabular}

TABLE 2: Demographic characteristics and primary surgeries

‡: Eta-Squared (n2) was used to compare the Kruskal-Wallis one-way analysis of variance.

ENT - ear, nose and throat; OBGYN - obstetrics and gynecology; OMFS - oral and maxillofacial surgery; IQR - interquartile range

Table 3 shows a between-group comparison of all recorded bedside procedures. Overall, the frequency of bedside procedures was low in this sample of patients. The most common procedure performed for all groups was central line placement. The positive group had a significantly higher proportion of central line placements than the other two groups ( $\mathrm{p}<0.001)$, but this difference (effect size) is classified as "small". 


\section{Cureus}

\begin{tabular}{|c|c|c|c|c|c|c|}
\hline & Untested $(n=4096)$ & Negative $(n=1412)$ & Positive ( $n=39)$ & P-values & Cramér's V & Effect size \\
\hline Central line & 119 (2.91\%) & $54(3.82 \%)$ & $9(23.08 \%)$ & $<0.01$ & 0.096 & $=$ Small \\
\hline Chest tube placement & $36(0.88 \%)$ & $13(0.92 \%)$ & $0(0 \%)$ & 1.00 & 0.008 & $<$ Small \\
\hline Midline & $1(0.02 \%)$ & $3(0.21 \%)$ & $0(0 \%)$ & 1.00 & 0.031 & $<$ Small \\
\hline Pacemaker & $1(0.02 \%)$ & $2(0.14 \%)$ & $0(0 \%)$ & 1.00 & 0.022 & $<$ Small \\
\hline Placement of intracardiac defibrillator & $1(0.02 \%)$ & $0(0 \%)$ & $0(0 \%)$ & $\cdot \cdot$ & .. & $\cdot \cdot$ \\
\hline Placement of intracardiac pacemaker & $6(0.15 \%)$ & $1(0.07 \%)$ & $1(2.56 \%)$ & 1.00 & 0.054 & $<$ Small \\
\hline Pleural drainage endoscopy & $26(0.63 \%)$ & $12(0.85 \%)$ & $1(2.56 \%)$ & 1.00 & 0.022 & $<$ Small \\
\hline Thoracotomy & $5(0.12 \%)$ & $2(0.14 \%)$ & $0(0 \%)$ & 1.00 & 0.004 & $<$ Small \\
\hline Tracheostomy & $16(0.39 \%)$ & $10(0.71 \%)$ & $2(5.13 \%)$ & 0.40 & 0.058 & $<$ Small \\
\hline
\end{tabular}

TABLE 3: Bedside procedures

Of the three groups, the majority of the positive patients went to the ICU (69.23\%) with an average LOS of 18 days (Table 4 ), whereas $16.72 \%$ of the untested group and $21.25 \%$ of the negative group were admitted to the ICU. The LOS for the untested and negative groups was 2.62 and 2.94 days, respectively. The positive group differed significantly from the negative and untested group when comparing ICU admissions $(p<0.001)$ and ICU LOS ( $\mathrm{p}<0.001)$. There was no significant difference between the negative and untested groups when it came to ICU admissions $(\mathrm{p}=0.625)$ and ICU LOS $(\mathrm{p}=0.328)$. Non-invasive ventilation was defined as requiring the use of bilevel positive airway pressure (BiPAP) or continuous positive airway pressure (CPAP), while invasive ventilation was defined as endotracheal intubation. Overall, a higher percentage of patients who tested positive for COVID-19 required ventilators (Table 4). Forty-six percent of the patients in the positive group required invasive ventilation compared to $4.22 \%$ of patients in the untested group $(\mathrm{p}<0.001)$, and $8.85 \%$ in the negative group $(\mathrm{p}<0.001)$ (Table 4$)$. One patient required extracorporeal membrane oxygenation (ECMO) during their hospital admission in the negative group. The difference between the untested and negative groups was not statistically significant $(\mathrm{p}=0.152)$. There were also statistically significant differences between all three groups when it came to the use of noninvasive ventilators ( $p<0.01)$. According to the effect size measure, the between-group differences in magnitude can be described as "small". The median days on invasive ventilation for the positive group was 17.71 compared to 2.08 in the negative group and 2.10 in the untested group.

\begin{tabular}{|c|c|c|c|c|c|c|}
\hline & Untested $(n=4096)$ & Negative $(n=1412)$ & Positive $(n=39)$ & P-values & Cramér's V & Effect size \\
\hline Admitted to ICU & $685(16.72 \%)$ & $300(21.25 \%)$ & $27(69.23 \%)$ & $<0.01$ & 0.122 & $=$ Small \\
\hline ICU length of stay, days - median (IQR) & $2.62(0-6.79)$ & $2.94(0-8.53)$ & $18.21(0-46.14)$ & $<0.01$ & $0.005^{\ddagger}$ & $<$ Small \\
\hline Invasive ventilation & $173(4.22 \%)$ & $125(8.85 \%)$ & $18(46.15 \%)$ & $<0.01$ & 0.171 & $=$ Small \\
\hline Noninvasive ventilation & $117(2.86 \%)$ & $68(4.82 \%)$ & $7(17.95 \%)$ & $<0.01$ & 0.081 & $=$ Small \\
\hline Days on noninvasive ventilator - median (IQR) & $3.21(0-12.83)$ & $2.99(0-9.58)$ & $5.75(0-32.42)$ & 1.00 & $0.009^{\ddagger}$ & $<$ Small \\
\hline Days on invasive ventilator - median (IQR) & $2.08(0-6.27)$ & $2.10(0-8.37)$ & $17.71(0-53.10)$ & 0.02 & $0.044^{\ddagger}$ & $<$ Small \\
\hline Extracorporeal membrane oxygenation & $0(0 \%)$ & $1(0.23 \%)$ & $0(0 \%)$ & & & \\
\hline
\end{tabular}

\section{TABLE 4: Intensive care unit usage}

‡: Eta-Squared (n2) was used to compare the Kruskal-Wallis one-way analysis of variance.

ICU - intensive care unit; IQR - interquartile range

The most common comorbidities associated with the positive group listed in descending order were a history of smoking, anemia, diabetes, and hypertension (Table 5). In both the untested and negative groups, the two most common comorbidities were a history of smoking and hypertension (Table 5). There was a significantly 
higher proportion of patients diagnosed with anemia in the positive group than in the negative $(p<0.001)$ and untested $(p<0.001)$ groups. There was no significant difference between the negative and untested groups ( $\mathrm{p}=0.163)$. These differences were "small" in magnitude $(\mathrm{V}=0.075)$.

\begin{tabular}{|c|c|c|c|c|c|c|}
\hline & Untested ( $n=4096)$ & Negative $(n=1412)$ & Positive (n=39) & P-values & Cramér's V & Effect size \\
\hline Any comorbidity & $1440(35.16 \%)$ & $500(35.41 \%)$ & $20(51.28 \%)$ & 1.00 & 0.028 & $<$ Small \\
\hline Alcohol abuse & $43(1.05 \%)$ & $20(1.42 \%)$ & $0(0 \%)$ & 1.00 & 0.018 & $<$ Small \\
\hline Anemia & $180(4.40 \%)$ & $75(5.31 \%)$ & $9(23.08 \%)$ & $<0.01$ & 0.075 & $=$ Small \\
\hline Cancer & 42 (1.03\%) & $12(0.85 \%)$ & $1(2.56 \%)$ & 1.00 & 0.015 & $<$ Small \\
\hline Cardiac disease & 37 (0.90\%) & $23(1.63 \%)$ & $1(2.56 \%)$ & 1.00 & 0.033 & $<$ Small \\
\hline Chronic pulmonary disease & $162(3.96 \%)$ & $72(5.10 \%)$ & $6(15.38 \%)$ & 0.17 & 0.052 & $<$ Small \\
\hline Congestive heart failure & $128(3.13 \%)$ & $48(3.40 \%)$ & $5(12.82 \%)$ & 1.00 & 0.046 & $<$ Small \\
\hline Depression & $155(3.78 \%)$ & $62(4.39 \%)$ & $3(7.69 \%)$ & 1.00 & 0.021 & $<$ Small \\
\hline Diabetes & $238(5.81 \%)$ & $95(6.73 \%)$ & $8(20.51 \%)$ & 0.16 & 0.053 & $<$ Small \\
\hline Hypertension & $534(13.04 \%)$ & $227(16.08 \%)$ & $8(20.51 \%)$ & 0.48 & 0.042 & $<$ Small \\
\hline Hypothyroidism & $162(4.46 \%)$ & $63(4.46 \%)$ & $1(2.56 \%)$ & 1.00 & 0.013 & $<$ Small \\
\hline Liver disease & $30(0.78 \%)$ & $7(1.63 \%)$ & $2(5.13 \%)$ & 0.76 & 0.044 & $<$ Small \\
\hline Malignancy & $32(0.78 \%)$ & $7(0.50 \%)$ & $2(5.13 \%)$ & 1.00 & 0.046 & $<$ Small \\
\hline Obesity & $166(4.05 \%)$ & $78(5.52 \%)$ & $4(10.26 \%)$ & 0.80 & 0.039 & $<$ Small \\
\hline Peripheral vascular disease & $85(2.08 \%)$ & $31(2.20 \%)$ & $3(7.69 \%)$ & 1.00 & 0.032 & $<$ Small \\
\hline Renal failure & $142(3.47 \%)$ & $60(4.25 \%)$ & $4(10.26 \%)$ & 1.00 & 0.034 & $<$ Small \\
\hline Rheumatoid arthritis & 53 (1.29\%) & $16(1.13 \%)$ & $0(0 \%)$ & 1.00 & 0.011 & $<$ Small \\
\hline Any smoking & $851(20.78 \%)$ & 256 (18.13\%) & $10(25.64 \%)$ & 1.00 & 0.031 & $<$ Small \\
\hline
\end{tabular}

TABLE 5: Comorbidities

When examining the pharmaceutical treatments of patients infected with COVID-19, antibiotics were used the most in all three groups. Patients who tested positive for COVID-19 required a higher proportion of antibiotics than patients who tested negative $(\mathrm{p}<0.001)$ and the untested patients $(\mathrm{p}<0.001)$. Additionally, more patients who tested negative required antibiotics than patients who were not tested $(p<0.01)$. A higher percentage of patients in the positive group than in the negative $(\mathrm{p}=0.01)$ and untested $(\mathrm{p}<0.001)$ received antivirals (Table 6). There was also a significant difference in glucocorticoids between the untested and negative group $(\mathrm{p}<0.001)$. These differences were "small" in magnitude.

\begin{tabular}{|c|c|c|c|c|c|c|}
\hline & Untested $(n=4096)$ & Negative $(n=1412)$ & Positive $(n=39)$ & P-values & Cramér's V & Effect size \\
\hline Antibiotics & $1319(32.21 \%)$ & $512(36.26 \%)$ & 32 (82.05\%) & 0.008 & 0.094 & $=$ Small \\
\hline Antivirals & 117 (2.86\%) & $70(4.96 \%)$ & $5(12.82 \%)$ & 0.008 & 0.066 & $=$ Small \\
\hline Glucocorticoids & 867 (21.17\%) & 384 (27.20\%) & 15 (38.46\%) & 0.008 & 0.070 & $=$ Small \\
\hline Immunoglobulin & $28(0.68 \%)$ & 22 (1.56\%) & $0(0 \%)$ & 0.008 & 0.041 & $<$ Small \\
\hline
\end{tabular}

TABLE 6: Medication use

The most common symptoms in the positive group were fever (33.33\%), cough (16.38\%), and shortness of breath (12.82\%). In the untested group, symptoms included fever (9.18\%), chills (3.88\%), and cough (3.78\%) 


\section{Cureus}

(Table 7). In the negative group, results showed fever (10.34\%), cough (7.37\%), and chills (3.54\%). A higher percentage of patients in the positive group had a fever than in both negative $(p<0.001)$ and untested $(\mathrm{p}<0.001)$ groups. There was no significant difference between negative and untested groups. There was a significant difference in cough occurrence between all three groups $(\mathrm{p}<0.01)$. There was no significant difference in the presence of cough as a symptom between negative and positive patients $(\mathrm{p}=0.113)$. There were significant differences between all three groups in the presence of "shortness of breath" ( $<<0.01)$, though the difference between the negative and positive groups was barely significant $(\mathrm{p}<0.01)$. These differences were "small" in magnitude.

\begin{tabular}{|c|c|c|c|c|c|c|}
\hline & Untested ( $n=4096$ ) & Negative $(n=1412)$ & Positive ( $n=39$ ) & P-values & Cramér's V & Effect size \\
\hline Abdominal pain & $65(1.59 \%)$ & $15(1.06 \%)$ & $0(0 \%)$ & 1.00 & 0.022 & $<$ Small \\
\hline Acute respiratory failure & $2(0.05 \%)$ & $0(0 \%)$ & $0(0 \%)$ & & & \\
\hline Chest pain & $51(1.25 \%)$ & $17(1.20 \%)$ & $0(0 \%)$ & 1.00 & 0.010 & $<$ Small \\
\hline Chills & $159(3.88 \%)$ & $50(3.54 \%)$ & $0(0 \%)$ & 1.00 & 0.018 & $<$ Small \\
\hline Cough & $155(3.78 \%)$ & $104(7.37 \%)$ & $6(16.38 \%)$ & $<0.01$ & 0.084 & $=$ Small \\
\hline Diarrhea & $27(0.66 \%)$ & 10 (0.71\%) & $0(0 \%)$ & 1.00 & 0.007 & $<$ Small \\
\hline Dyspnea & $47(1.15 \%)$ & $22(1.56 \%)$ & $2(5.13 \%)$ & 1.00 & 0.033 & $<$ Small \\
\hline Emesis & $118(2.88 \%)$ & $37(2.62 \%)$ & $0(0 \%)$ & 1.00 & 0.016 & $<$ Small \\
\hline Fatigue & $15(0.37 \%)$ & $3(0.21 \%)$ & $2(5.13 \%)$ & 0.51 & 0.068 & $<$ Small \\
\hline Fever & $376(9.18 \%)$ & $146(10.34 \%)$ & $13(33.33 \%)$ & $<0.01$ & 0.070 & $<$ Small \\
\hline Malaise & $1(0.02 \%)$ & $1(0.07 \%)$ & $0(0 \%)$ & 1.00 & 0.011 & $<$ Small \\
\hline Myalgia & $1(0.02 \%)$ & $2(0.14 \%)$ & $0(0 \%)$ & 1.00 & 0.022 & $<$ Small \\
\hline Other pain & 53 (1.29\%) & 10 (0.71\%) & $1(2.56 \%)$ & 1.00 & 0.026 & $<$ Small \\
\hline Palpitations & $16(0.39 \%)$ & $6(0.42 \%)$ & $0(0 \%)$ & 1.00 & 0.006 & $<$ Small \\
\hline Shortness of breath & $80(1.95 \%)$ & $47(3.33 \%)$ & $5(12.82 \%)$ & $<0.01$ & 0.070 & $<$ Small \\
\hline
\end{tabular}

TABLE 7: Symptoms

One-hundred percent of the patients in the untested group, $94.12 \%$ of the negative group, and $79.49 \%$ of the positive group were discharged from the hospital (Table 8 ). These differences are statistically significant $(\mathrm{p}<0.01)$. At the time of this study, some patients remain hospitalized. The mortality rate in the positive group was $7.69 \%$ compared to only $1.10 \%$ in the untested group and $1.56 \%$ in the positive group, though these differences were not statistically significant. The LOS was found to be higher in the positive population with a median LOS of 15.42 days compared to 2.38 days in the untested group and 2.46 days in the negative group, but these differences were not statistically significant. When comparing 30-day readmission rates, the positive group had the highest at $10.26 \%$, the untested group was $7.76 \%$, and the negative group was $6.73 \%$; these differences were statistically significant $(\mathrm{p}<0.01)$. The discharge disposition is listed below in Table 8 . The most common discharge was to home with $70.07 \%$ in the untested population, $63.39 \%$ in the negative group, and $30.77 \%$ in the positive group. Home discharge proportions differed significantly between all three groups ( $\mathrm{p}<0.001$ ). All the differences were "small" in magnitude (Table 8 ). 


\section{Cureus}

\begin{tabular}{|c|c|c|c|c|c|c|}
\hline & Untested ( $n=4096)$ & Negative $(n=1412)$ & Positive $(n=39)$ & P-values & Cramér's V & Effect size \\
\hline Discharged & $4069(99.34 \%)$ & $1329(94.12 \%)$ & $31(79.49 \%)$ & $<0.01$ & 0.190 & $=$ Small \\
\hline Expired & $45(1.10 \%)$ & $22(1.56 \%)$ & $3(7.69 \%)$ & 0.52 & 0.052 & $<$ Small \\
\hline Total length of stay, days - median (IQR) & $2.38(0-5.96)$ & $2.46(0-6.79)$ & $15.42(0-32.80)$ & 1.00 & $0.010^{\ddagger}$ & $=$ Small \\
\hline Readmitted & $318(7.76 \%)$ & $95(6.73 \%)$ & $4(10.26 \%)$ & $<0.01$ & 0.192 & $=$ Small \\
\hline \multicolumn{7}{|l|}{ Discharge disposition } \\
\hline Left against advice & $26(0.63 \%)$ & $7(0.50 \%)$ & $1(2.56 \%)$ & 1.00 & 0.022 & $<$ Small \\
\hline Expired & $46(1.12 \%)$ & $22(1.56 \%)$ & $3(7.69 \%)$ & 0.52 & 0.051 & $<$ Small \\
\hline Home & $2870(70.07 \%)$ & $895(63.39 \%)$ & $12(30 . / / \%)$ & $<0.01$ & 0.092 & $=$ Small \\
\hline Home with home health service & $477(11.65 \%)$ & $163(11.54 \%)$ & $3(7.69 \%)$ & 1.00 & 0.010 & $<$ Small \\
\hline Hospice & $45(1.10 \%)$ & $18(1.27 \%)$ & $3(7.69 \%)$ & 1.00 & 0.051 & $<$ Small \\
\hline Other hospital & $12(0.29 \%)$ & $2(0.14 \%)$ & $0(0 \%)$ & 1.00 & 0.014 & $<$ Small \\
\hline Remanded to law enforcement & $6(0.15 \%)$ & $4(0.28 \%)$ & $0(0 \%)$ & 1.00 & 0.015 & $<$ Small \\
\hline Long term care facility & $38(0.93 \%)$ & $22(1.56 \%)$ & $2(5.13 \%)$ & 0.99 & 0.041 & $<$ Small \\
\hline Skilled nursing facility & $381(9.30 \%)$ & $112(7.93 \%)$ & $4(10.26 \%)$ & 1.00 & 0.021 & $<$ Small \\
\hline Psychiatric facility & $6(0.15 \%)$ & $2(0.14 \%)$ & $0(0 \%)$ & 1.00 & 0.003 & $<$ Small \\
\hline Rehabilitation & $160(3.91 \%)$ & $73(5.17 \%)$ & $2(5.13 \%)$ & 1.00 & 0.028 & $<$ Small \\
\hline Unknown & $29(0.71 \%)$ & $92(6.52 \%)$ & $9(23.08 \%)$ & $<0.01$ & 0.203 & $=$ Small \\
\hline
\end{tabular}

\section{TABLE 8: Discharge and length of stay}

‡: Eta-Squared (n2) was used to compare the Kruskal-Wallis one-way analysis of variance.

IQR - interquartile range

Acute kidney failure and cardiac complications were the most frequently reported complications among all three groups (Table 9). The only statistically significant difference between groups was in the proportion of patients with pulmonary complications $(p<0.001)$, with positive patients having the highest proportion. Effect size assessment revealed no differences in magnitude between the three groups that can be classified as greater than "small". 


\begin{tabular}{|c|c|c|c|c|c|c|}
\hline & Untested $(n=4096)$ & Negative $(n=1412)$ & Positive $(n=39)$ & P-values & Cramér's V & Effect size \\
\hline Acute kidney failure & $416(10.16 \%)$ & $168(11.9 \%)$ & 12 (30.77\%) & 0.02 & 0.060 & $<$ Small \\
\hline Cardiac complications & $377(9.2 \%)$ & $142(10.06 \%)$ & $4(10.26 \%)$ & 1.00 & 0.013 & $<$ Small \\
\hline Infection & $1(0.02 \%)$ & $2(0.14 \%)$ & $0(0 \%)$ & 1.00 & 0.022 & $<$ Small \\
\hline Pulmonary complications & $48(1.17 \%)$ & $39(2.76 \%)$ & $5(12.82 \%)$ & $<0.01$ & 0.091 & $=$ Small \\
\hline SIRS and septic shock & $127(3.1 \%)$ & $56(3.97 \%)$ & $6(15.38 \%)$ & 0.08 & 0.059 & $<$ Small \\
\hline Urinary incontinence & $31(0.76 \%)$ & $14(0.99 \%)$ & $0(0 \%)$ & 1.00 & 0.014 & $<$ Small \\
\hline Urinary injury & $11(0.27 \%)$ & $1(0.07 \%)$ & $0(0 \%)$ & 1.00 & 0.019 & $<$ Small \\
\hline Urinary tract infection & $94(2.29 \%)$ & $43(3.05 \%)$ & $3(7.69 \%)$ & 1.00 & 0.035 & $<$ Small \\
\hline
\end{tabular}

\section{TABLE 9: Complications}

SIRS - systemic inflammatory response syndrome

\section{Discussion}

To our knowledge, this is the first paper to investigate both the tested and untested patient populations of all urgent and emergent surgeries performed in a regional healthcare system during the COVID-19 pandemic. All currently available data suggests that SARS-CoV-2 infection can lead to prolonged ICU care, increased ventilator requirements, and a higher mortality rate following surgery $[4,14,15]$. The aim of this study was to examine 5,547 patients from eight hospitals who underwent urgent and emergent surgeries from March 1, 2020 to May 17, 2020. The patients were divided into three groups: untested for COVID-19, COVID-19 positive, and COVID-19 negative patients. Their clinical outcomes and characteristics were compared.

In our study, which is the largest published cohort of its kind to date, the untested population was found to have similar outcomes to those who tested negative for COVID-19. At the time this data was collected, all patients were not routinely tested for the virus. Testing was performed on symptomatic patients (febrile, cough, respiratory distress, etc.) postoperatively since these patients came in with urgent or emergent issues requiring immediate procedures.

It is important to note that we decided to present our findings using both effect sizes (Cramer's V) and adjusted p-values because it describes the magnitude and probability of the between-group differences more appropriately, especially when comparing groups with such large sample size differences. Methodological flaws related to the utilization of unadjusted p-values with large sample sizes have been widely covered in other publications [16].

One-hundred percent of the untested population was discharged from the hospital compared to $94.1 \%$ of negative patients and $79.5 \%$ of the positive group. At the time of this manuscript, some patients were still admitted; thus, outcomes could not be reached on all of the cohort. This fact may skew some of the results, such as the ventilator time and LOS, since there may be patients that are still being ventilated in the ICU with the potential for further complications; however, the majority of patients have a record of a clinical outcome making this a good representation of the cohorts.

When comparing this paper to other contemporary studies, the mortality rate was $7.69 \%$ in the COVID-19 positive patients. This is a lower rate than what was reported by COVIDSurg Collaborative $23.8 \%$, Lei and colleagues $20.5 \%$, and $50 \%$ mentioned by Aminian and colleagues $[4,9,11]$. The LOS was found to be similar between the COVIDSurg Collaborative and our study. The LOS in our study was 15.42 days, and the COVIDSurg Collaborative study reported a LOS of 16 days in their emergency surgery group. It is important to note that the COVIDSurge Collaborative had missing invasive ventilator data on 916 out of 1,115 patients. In our study, rather than dividing the duration of ventilation into categorical groups, with " $\geqslant 72$ " as the longest category, we tracked the actual number of days on an invasive ventilator; thus, it is difficult to compare our study to the COVIDSurge Collaborative directly. The median number of days on invasive ventilator support for COVID-19 positive patients in our study was 17.71 days. Additionally, as opposed to $18.2 \%$ of patients in our study, $34.3 \%$ of patients in studies of COVIDSurg Collaborative and $44.1 \%$ of patients in Lei et al. were admitted to the ICU.

A study by Zhou and colleagues investigated risk factors associated with increased mortality from COVID19. Older age was found to have an odds ratio of 1.10 , with a median age of 69 for in-hospital mortality and 
comorbidities such as hypertension and diabetes [17]. Wu and colleagues found that patients with comorbidities such as hypertension and diabetes were more likely to develop acute respiratory distress syndrome (ARDS) and older patients were more likely to progress from ARDS to death (hazard ratio 3.26) [18]. They classified older age as 65 or older. The most common comorbidities associated with our COVID-19 positive group listed in descending order were a history of smoking, anemia, diabetes, and hypertension. Our study had diabetes and hypertension in common with the above studies, whereas none of the studies evaluated for a past medical history of anemia. Only Wu et al. examined smoking history in their study, but the rate was much lower - $6 \%$ versus $25.64 \%$ in our positive group [18].

A systematic review of 43 clinical symptoms showed that the most common symptoms were fever (83.3\%), cough (60.3\%), and fatigue (38.0\%) [19]. The most common symptoms of the COVID-19 positive group in our study were fever (33.33\%), cough (16.38\%), and shortness of breath (12.82\%). Our study also identified fever and cough as the most prevalent symptoms for the positive group, but they were in lower percentages.

Lei et al. complication rates included ARDS (32.4\%), shock (29.4\%), secondary infection (29.4\%), arrhythmia (23.5\%), acute cardiac injury (14.7\%), and acute kidney injury (5.9\%) [4]. The COVIDSurg Collaborative study found that $51.2 \%$ of patients had pulmonary complications [11]. Our positive group's most common complications were acute kidney injury (30.77\%), systemic inflammatory response syndrome (SIRS), septic shock (15.38\%), and pulmonary complications (12.82\%). Therefore, our pulmonary complications were significantly lower than the COVIDSurg Collaborative study.

General and orthopedic surgeries were the most common primary surgeries performed. This is similar to what was reported in the COVIDSurg Collaborative study, with their top two primary surgeries being gastrointestinal and general surgery and orthopedics [11].

As hospitals began to reopen for elective surgery in the Denver and Wichita areas, new protocols were created. Currently, all patients are required to have a preoperative COVID-19 test performed. After being tested, all patients are to self-quarantine prior to the planned procedure. During that time, a daily temperature log must be kept. Only the patients who have a negative test are cleared for surgery. As stated above, the patients included in this study were only tested if they were symptomatic postoperatively. There is a possibility that some patients in the untested population had COVID-19 but were asymptomatic. In a recent study of 78 patients who tested positive for COVID-19 in Wuhan, China, 33 (42\%) were asymptomatic [20]. Additionally, recent studies also suggest that the sensitivity and specificity of reverse transcriptionpolymerase chain reaction (RT-PCR) tests improve when patients actually start experiencing symptoms and are likely worse in the asymptomatic individuals or in the asymptomatic disease stage [21, 22]. Therefore, since the untested group had the least amount of admissions to the ICU, less time on the ventilator, and all the patients were discharged from the hospital, it raises the question: do we need to test everyone?

New COVID-19 protocols require many resources to test patients: staff to collect the samples, labs to run the samples, and staff to monitor results. Surgeries may even be canceled for COVID-19 positive asymptomatic patients. These recommendations add to the financial and social burden to both the patient and the hospital where the procedure is to be performed. This group of hospitals spends $\$ 11,000$ a day on tests. That doesn't include the cost in terms of OR protocols, personal protective equipment (PPE), and staff safety. In our study, the untested group had better outcomes than the negative group. Therefore, the question remains if the expense and the resource utilization is worth it if the presumptive asymptomatic COVID-19 positive patients do not have negative clinical results. Therefore, we recommend stratifying perioperative risk for patients and improving the cost in terms of perioperative testing moving forward with elective surgery.

\section{Conclusions}

We propose, as an alternative, to create criteria to select asymptomatic patients for testing. Patients with comorbidities that have been shown to increase mortality rate (hypertension, diabetes, etc.) would be tested preoperatively. In addition, we could also rationalize testing patients that are older than 65 since they have an increased risk, as discussed above. Testing could also be stratified based on the type of surgical procedure performed, pending better outcomes data on individual surgeries. This approach would be another alternative to help save scarce resources instead of testing every patient preoperatively going forward. We understand as a precaution testing every patient or treating patients as a presumed positive is important at this time to prevent spread. Our goal is to start discussing a plan for going forward, and we understand that this plan won't be put into full effect until we have either heard immunity or a vaccine in place to protect staff and patients from the asymptomatic spread. We believe our study opens the door to further studies that look at outcomes of asymptomatic COVID-19 patients who test positive postoperatively, and we plan to investigate these outcomes in the future.

\section{Additional Information}

\section{Disclosures}

Human subjects: Consent was obtained by all participants in this study. HCA HealthCare C.A.R.R.I.E. Submission issued approval 2020-285. IRB Exemption per 45CFR46.102(l) OR c) engaging in research with 
sufficient human subject protections in the design to meet one or more IRB exemption criteria set forth in 45CFR46.104. Animal subjects: All authors have confirmed that this study did not involve animal subjects or tissue. Conflicts of interest: In compliance with the ICMJE uniform disclosure form, all authors declare the following: Payment/services info: All authors have declared that no financial support was received from any organization for the submitted work. Financial relationships: All authors have declared that they have no financial relationships at present or within the previous three years with any organizations that might have an interest in the submitted work. Other relationships: All authors have declared that there are no other relationships or activities that could appear to have influenced the submitted work.

\section{Acknowledgements}

Megan Novak for editing and reformatting our study for publication.

\section{References}

1. Gandhi M, Yokoe DS, Havlir DV: Asymptomatic transmission, the Achilles' heel of current strategies to control Covid-19. N Engl J Med. 2020, 382:2158-2160. 10.1056/NEJMe2009758

2. Shakiba M, Nazari SSH, Mehrabian F, et al.: Seroprevalence of COVID-19 virus infection in Guilan province, Iran [PREPRINT]. BMJ. 2020, 10.1101/2020.04.26.20079244

3. Maida FD, Antonelli A, Porreca A, Rocco B, Mari A, Minervini A : Clinical characteristics and outcomes of patients undergoing surgeries during the incubation period of COVID-19 infection. EClinicalMedicine. 2020, 22:100362. 10.1016/j.eclinm.2020.100362

4. Lei S, Jiang F, Su W, et al.: Clinical characteristics and outcomes of patients undergoing surgeries during the incubation period of COVID-19 infection. EClinicalMedicine. 2020, 21:100331. 10.1016/i.eclinm.2020.100331

5. The ASA and APSF joint statement on perioperative testing for the COVID-19 virus . (2020). https://www.asahq.org/about-asa/newsroom/news-releases/2020/04/asa-and-apsf-joint-statement-onperioperative-testing-....

6. Morris M, Pierce A, Carlisle B, Vining B, Dobyns J: Pre-operative COVID-19 testing and decolonization. Am J Surg. 2020, 220:558-560. 10.1016/j.amjsurg.2020.05.027

7. Stahel PF: How to risk-stratify elective surgery during the COVID-19 pandemic? . Patient Saf Surg. 2020, 14:8. 10.1186\%2Fs 13037-020-00235-9

8. Kuy SR, Gupta R, Correa R, et al.: Best practices for a Covid-19 preparedness plan for health systems . NEJM Catalyst. 2020,

9. Aminian A, Safari S, Razeghian-Jahromi A, et al.: COVID-19 outbreak and surgical practice. Ann Surg. 2020, 272:27-29. 10.1097/SLA.0000000000003925

10. Liang W, Guan W, Chen R, et al.: Cancer patients in SARS-CoV-2 infection: a nationwide analysis in China . Lancet Oncol. 2020, 21:335-337. 10.1016/S1470-2045(20)30096-6

11. COVIDSurg Collaborative: Mortality and pulmonary complications in patients undergoing surgery with perioperative SARS-CoV-2 infection: an international cohort study. Lancet. 2020, 396:27-38. 10.1016\%2FS0140-6736(20)31182-X

12. Cohen J: Statistical power analysis for the behavioral sciences, 2nd edition . Lawrence Erlbaum Associates, Hillsdale; 1988.

13. Abdi H: Holm's sequential Bonferroni procedure. Encyclopedia of research design. Salkind NJ (ed): SAGE Publications, Inc., Thousand Oaks; 2010. 1:574-577. 10.4135/9781412961288.n178

14. Möhlenkamp S, Thiele H: Ventilation of COVID-19 patients in intensive care units . Herz. 2020, 45:329-331. 10.1007\%2Fs00059-020-04923-1

15. Meng L, Qiu H, Wan L, et al.: Intubation and ventilation amid the COVID-19 outbreak. Anesthesiology. 2020, 132:1317-1332. 10.1097/ALN.0000000000003296

16. Halsey LG: The reign of the p-value is over: what alternative analyses could we employ to fill the power vacuum?. Biol Lett. 2019, 15:20190174. 10.1098/rsbl.2019.0174

17. Zhou F, Yu T, Du R, et al.: Clinical course and risk factors for mortality of adult inpatients with COVID-19 in Wuhan, China: a retrospective cohort study. Lancet. 2020, 395:1054-1062. 10.1016/S01406736(20)30566-3

18. Wu C, Chen X, Cai Y, et al.: Risk factors associated with acute respiratory distress syndrome and death in patients with coronavirus disease 2019 pneumonia in Wuhan, China. JAMA Intern Med. 2020, 180:934-943. 10.1001/jamainternmed.2020.0994

19. Fu L, Wang B, Yuan T, et al.: Clinical characteristics of coronavirus disease 2019 (COVID-19) in China: a systematic review and meta-analysis. J Infect. 2020, 80:656-665. 10.1016\%2Fj.jinf.2020.03.041

20. Yang R, Gui X, Xiong Y: Comparison of clinical characteristics of patients with asymptomatic vs symptomatic coronavirus disease 2019 in Wuhan, China. JAMA Network Open. 2020, 3:e2010182. 10.1001/jamanetworkopen.2020.10182

21. Sethuraman N, Jeremiah SS, Ryo A: Interpreting diagnostic tests for SARS-CoV-2 . JAMA. 2020, 323:22492251. 10.1001/jama.2020.8259

22. Watson J, Whiting PF, Brush JE: Interpreting a Covid-19 test result. BMJ. 2020, 369:m1808. May 12369 